\title{
TÉCNICA DE HIGIENIZAÇÃO SIMPLES DAS MÃOS: A PRÁTICA ENTRE ACADÊMICOS DA ENFERMAGEM
}

\author{
TECHNIQUE OF THE SIMPLE HANDS WASHING: THE PRACTICAL \\ BETWEEN NURSING ACADEMICS
}

\section{TÉCNICA DE HIGIENIZACIÓN SIMPLE DE MANOS: LA PRÁCTICA ENTRE ACADÉMICOS DE ENFERMERÍA}

\author{
Anaclara Ferreira Veiga Tipple ${ }^{*}$ \\ Aliny Santana de SA $^{* *}$ \\ Katiane Martins MendonçA ${ }^{* * *}$ \\ Adenícia Custódia SiLVa e Sousa ${ }^{* * * *}$ \\ Silvana de Lima Vieira dos Santos ${ }^{* * * * *}$
}

\begin{abstract}
RESUMO
Estudo realizado com graduandos de enfermagem do último ano/semestre de Instituições de Ensino Superior (IES) em Goiás no período de 2005 e 2006, com objetivo de avaliar a técnica de Higienização das Mãos (HM) descrita por graduandos de enfermagem e identificar a contribuição das IES na formação do aluno sobre HM. Todos os aspectos éticos foram atendidos. Os dados foram obtidos por meio da aplicação de questionário, previamente avaliado, e processados no programa Epiinfo. Participaram do estudo 286 acadêmicos, sendo que 132 do ano de 2005 e 154 de 2006. Apesar de afirmarem ter conhecimento teórico com relação à técnica de HM apresentaram baixo desempenho ao descrevê-la. Os resultados mostram que a abordagem dada pelas IES com relação à técnica de HM, não tem proporcionado sedimentação do conhecimento que resultasse na execução correta da técnica de higienização das mãos.
\end{abstract}

Palavras chave: Lavagem de mãos, controle de infecções, ensino, cuidados de enfermagem.

\begin{abstract}
Study that was carried out with the last year/semester of higher education institutions (HEI) in Goiás in the period 2005 to 2006, in order to evaluate the hands washing (HW) the technique as described by the nursing students and identify the contribution of IES in the education of the student on HW. All ethical aspects were observed. Data collection were obtained through the application of a questionnaire, previously evaluated and processed in the Epiinfo. Study participants were conformed by 286 academics, with 132 during 2005 and 154 during 2006. Despite claiming to have known theoretical aspects about the technical performance of HW, it was not enough to describe it. Results show that the approach given by HEI as far as the technique of HW, is concerned, have not provided the knowledge sedimentation that result on correct execution of the hands washing technique.
\end{abstract}

Key words: Hands washing, control of infections, teaching, nursing care.

\footnotetext{
* Professora adjunto da Faculdade de Enfermagem/Universidade Federal de Goiás. E-mail: anaclara@fen.ufg.br

** Acadêmica de enfermagem. Bolsista Voluntária de Iniciação Cientifica 2006-2007, veiculada ao Núcleo de Estudos e Pesquisas em Infecção Hospitalar - NEPIH/Faculdade de Enfermagem/ Universidade Federal de Goiás. E-mail:alinysantana@ yahoo.com.br

${ }^{* * *}$ Enfermeira. Mestranda pela Faculdade de Enfermagem/Universidade Federal de Goiás. E-mail: katiane2303@gmail.com

${ }^{* * * *}$ Professora adjunto da Faculdade de Enfermagem/Universidade Federal de Goiás. E-mail:adenicia@fen.ufg.br

${ }^{* x+* * *}$ Professora da Faculdade de Enfermagem/Universidade Federal de Goiás. E-mail: silvanalvs@hotmail.com
} 


\section{RESUMEN}

Estudio realizado con estudiantes de enfermería en el último año/semestre de Instituciones de Educación Superior (IES) en Goiás en el período comprendido entre 2005 y 2006, para evaluar la técnica de lavado de las manos (LM) descrita por los estudiantes de enfermería y determinar la contribución de la IES en la formación del estudiante en LM. Se cautelaron todos los aspectos éticos. Los datos se obtuvieron mediante la aplicación de un cuestionario, previamente evaluado y tratados en el Epiinfo. Los participantes del estudio fueron 286 académicos, con 132 del año 2005 y 154 de 2006. A pesar de que afirman haber tenido conocimiento teórico con respecto a las prestaciones técnicas de LM, éste era insuficiente para describirlo. Los resultados muestran que el enfoque dado por el IES en lo que respecta a la técnica de LM, no ha proporcionado la sedimentación de los conocimientos que puedan llevar a la ejecución correcta de la técnica de lavado de manos.

Palabras clave: Lavado de manos, control de infección, enseñanza, atención de enfermería.

Fecha recepción: 03/11/08 Fecha aceptación: 16/11/09

\section{INTRODUÇÃO}

As mãos possuem a capacidade de abrigar microrganismos e transferi-los de uma superfície a outra. Assim, a adoção de medidas como a Higienização das Mãos (HM) com a água e sabão líquido ou pelo uso do álcool a $70 \%$ possui comprovadamente alta eficácia na prevenção e controle de infecções $(1,2)$. A eficácia desta prática foi comprovada por vários estudos $(1,3,4)$.

Os Centers for Disease Control and Prevention (CDC) publicaram em outubro de 2002, o Guideline de Higienização das Mãos, no qual o termo "lavagem de mãos" foi substituído por "higienização das mãos". O termo engloba a higienização simples das mãos, a higienização anti-séptica, a fricção de antiséptico (uso de preparações alcoólicas) e a anti-sepsia cirúrgica (5).

Em 1989, o Ministério da Saúde editou o Manual "Lavar as mãos" com a finalidade de promover a normatização do procedimento, considerado pouco valorizado nos estabelecimentos de saúde (6). Este manual descreve passo a passo a técnica de HM que só em 2007 volta a ser enfatizada no manual de $\mathrm{HM}$ publicado pela Agência Nacional de Vigilância Sanitária (ANVISA) em 2007 (3) e em 2008 (4). A importância dessa medida é reconhecida no anexo IV da Portaria 2616/98, ainda em vigência, que possui instruções sobre o Programa de Controle de Infecções Hospitalares nos Estabelecimentos de Assistência à Saúde (EAS) no país (7).

$\mathrm{O}$ mais recente manual abordando a temática (4) apresenta as indicações para realização da HM, distinguindo-as de acordo com o uso de água e sabão (quando as mãos estiverem visivelmente sujas ou contaminadas com sangue e outros fluidos corporais; ao iniciar e terminar o turno de trabalho; antes e após ir ao banheiro; fazer as refeições; preparar alimentos; manipular medicamentos; entrar em contato com paciente colonizado ou infectado por $C$. difficile e após várias aplicações consecutivas de produto alcoólico) e uso de preparações alcoólicas (antes e após contato com o paciente e remoção de luvas; antes de realizar procedimentos assistenciais e manipular dispositivos invasivos; antes de calçar luvas para inserção de dispositivos invasivos que não requeiram preparo cirúrgico; após risco de exposição a fluidos corporais; ao mudar de um sítio corporal contaminado, para outro, limpo, durante o cuidado ao paciente e após contato com objetos inanimados e superfícies imediatamente próximas ao paciente).

O mesmo manual (4) recomenda as seguintes etapas de desenvolvimento da técnica de higienização simples das mãos: retirar 
adornos, abrir a torneira e molhar as mãos, evitando encostar-se a pia; evitar água muito quente ou muito fria, a fim de prevenir o ressecamento da pele; aplicar sabão líquido na palma da mão em quantidade suficiente para cobrir todas as superfícies (seguir a quantidade recomendada pelo fabricante). Realizar a friç̧ão de todas as partes das mãos incluindo palmas, dorso, espaços interdigitais, dorso dos dedos, polegares, polpas digitais e punhos; enxaguá-las retirando os resíduos de sabão, no sentido dos dedos para os punhos, evitando contato direto das mãos ensaboadas com a torneira. Para finalizar, proceder a secagem com papel toalha descartável, iniciando pelas mãos e seguindo em direção aos punhos; posteriormente desprezar o papel tolha na lixeira destinada a resíduos comuns. A duração do procedimento depende do tipo, no caso da HM simples é de aproximadamente 40 a 60 segundos $(3,4)$.

Observamos na assistência a saúde atitudes e opiniões contraditórias, há uma valorização das inovações tecnológicas complexas, em detrimento de procedimentos básicos (embora não menos importantes) como é o caso da atenção que se deve ou que se deveria ter para com a HM.

Apesar da importância epidemiológica para a prevenção e controle de infecção, diversos autores têm demonstrado a falta de compromisso e de responsabilidade dos profissionais com esta prática, indicando a necessidade de repensar estratégias que repercutam na mudança de comportamento do profissional $(8,9)$.

Uma revisão bibliográfica com o objetivo de caracterizar os relatos científicos sobre $\mathrm{HM}$ e de contextualizar divergências entre a prática e o ideal preconizado, pesquisou publicações dos últimos 20 anos disponíveis na base de dados PubMed e verificou que existe a interferência do comportamento, do hábito, do ambiente e do contexto assistencial na baixa adesão a essa prática, bem como a necessidade de compreender e transformar a realidade, minimizando as divergências evidenciadas (10).

Estudos buscaram justificativas dos próprios Profissionais da Área da Saúde (PAS) para a não adesão a HM, dentre elas estão: sobrecarga de trabalho, falta de tempo e de recursos materiais, recursos materiais inadequados, falta de motivação, irresponsabilidade, falta de conscientização da equipe multiprofissional, pouca importância à contaminação cruzada, ausência de pias próximas ao paciente e reações cutâneas nas mãos $(1,8$, $11,12)$.

Um estudo de intervenção que utilizou diferentes estratégias de incentivo à $\mathrm{HM}$ e que foram construídas com a participação dos membros da equipe de saúde da instituição mostrou que não houve impacto significativo nos índices de adesão e que com o passar do tempo a tendência era de queda destes índices (13).

A enfermagem atua de forma direta e indireta na assistência e por representar, na maioria das instituições de saúde, o maior percentual de trabalhadores, faz-se imprescindível sua atuação de forma a prevenir e controlar infecções e neste contexto a HM tem um papel essencial $(11,14)$.

Alguns autores ressaltam a importância de agir e trabalhar o tema HM precocemente nos cursos de graduação, com a finalidade de promover a construção do hábito correto de HM $(12,14)$.

A formação dos profissionais com uma percepção de prevenção, talvez seja um dos grandes desafios que o ensino na área da saúde enfrenta (12). No caso da HM interessa não apenas a adesão, mas a sua execução de forma correta de onde se infere a necessidade de que a técnica seja enfatizada nos cursos de formação da área da saúde. Acreditamos que os resultados apresentados neste estudo servirão para reconhecimento das reais necessidades no que diz respeito ao ensino da técnica de HM e fatores envolvidos, sob uma visão de futuros profissionais de saúde. 


\section{OBJETIVOS}

- Avaliar a técnica de HM descrita por graduandos de enfermagem.

- Identificar a contribuição da Instituição de Ensino Superior (IES) na formação do aluno sobre HM.

\section{METODOLOGÍA}

Estudo descritivo cujos dados foram obtidos de duas formas.

1) Extraídos do banco de dados do Núcleo de Estudos e Pesquisa em Infecção Hospitalar (NEPIH) da Faculdade de Enfermagem FEN/UFG (12) que registrou informações acerca da HM de graduandos do último ano/ semestre dos cursos da área da saúde do Estado de Goiás no ano de 2005. Dentre 312 estudantes matriculados em cursos de graduação em enfermagem de cinco IES, 182 participaram do estudo.

Foi calculada uma amostra representativa com auxílio do Statistical, recurso estatístico do software Epiinfo versão 3.3 de 2004 (15), com índice de confiabilidade $95 \%$.

Estes dados foram coletados utilizandose um check list que foi construído contemplando os aspectos específicos da técnica e princípios da HM e seu ensino, aspectos não analisados no estudo desenvolvido por Tipple et al. (14).

2) Aplicação de questionário aos formandos de 2006 seguindo os mesmos passos metodológicos quando aplicado em 2005.
A coleta foi realizada em sala de aula após agendamento, no momento da sua aplicação foram esclarecidos os objetivos do trabalho e os alunos que aceitaram participar assinaram o termo de consentimento. Foi obtida a quantidade mínima de $54 \%$ de questionários respondidos em cada IES.

Dentre os cinco cursos participantes em 2005, um não consentiu em participar do estudo em 2006, desta forma foram excluídos os estudantes correspondentes a este curso em 2005. O questionário contemplou especificamente os dados referentes à técnica de HM e seu ensino nas IES.

A análise foi realizada com o auxílio da estatística descritiva, pela medida de freqüência das variáveis em estudo. Os dados foram processados no programa Epiinfo, versão 2004 (15) e apresentados em figuras e tabelas.

Atendendo a resolução número 196/96 (16), o projeto foi aprovado pelo Comitê de Ética em pesquisa da Universidade Federal de Goiás (protocolo número: 017/2005), os dados foram coletados após aquiescência das IES e dos sujeitos, aos quais foi garantido sigilo e o anonimato, por meio do termo de consentimento livre e esclarecido.

\section{RESULTADOS E DISCUSSÃO}

Participaram da pesquisa 286 acadêmicos, sendo que 132 graduandos de Enfermagem no Estado de Goiás do ano de 2005 e 154 do ano de 2006. Os graduandos foram questionados a respeito dos enfoques abordados com relação ao tema HM por suas respectivas IES, apresentado na Tabela 1. 
Tabela 1. Distribuição de freqüência e porcentagem dos enfoques relacionados à higienização das mãos as quais os graduandos de enfermagem participaram durante a graduação $(n=286)$. Goiás, 2005/2006.

\section{Enfoques abordados}

Técnica de higienização das mãos

Importância das mãos como veículo de contaminação cruzada

Recursos materiais indicados / necessários

Uso do álcool 70\%

$\begin{array}{cc}\text { Fr } & \text { \% } \\ 276 & 96,5 \\ 268 & 93,7 \\ 170 & 59,4 \\ 107 & 37,4\end{array}$

Chama a atenção o fato de apenas 107 $(37,4 \%)$ estudantes afirmarem conhecer informações sobre o uso de preparações alcoólicas. Estes produtos podem substituir a HM quando estas não apresentarem sujidade visível, em situações adversas $(1,5,13)$.

\section{A técnica de higienização das mãos}

Foi solicitado que os sujeitos descrevessem como realizavam a técnica de HM, questão que foi respondida por $258(90,2 \%)$ acadêmicos. Destes, somente $15(5,8 \%)$ enfatizaram a necessidade de retirar os fômites antes da realização desta técnica. No estudo realizado por Scheidt e Carvalho (9) com PAS também obtiveram um baixo índice de adesão (16,0\%) quanto à retirada de adornos antes da realização da técnica de HM o mesmo ocorreu no estudo realizado por Felix (17) entre acadêmicos de enfermagem, dos quais $64,6 \%$ não retiraram os fômites antes de executar os passos de HM.

Um estudo realizado em uma unidade de terapia intensiva com 60 enfermeiras, a análise multivariada dos fatores de risco mostrou que os anéis eram o único fator para carrear bacilos gram-negativos e $S$. aureus e que a concentração de microrganismos está relacionada com a quantidade de anéis utilizados (18).

Os fômites propiciam a permanência da microbiota transitória sob estes adereços, possibilitando a disseminação de micror- 
ganismos patogênicos, principalmente os gram-negativos (5). O que nos leva a questionar como este assunto tem sido discutido pelas IES. Estaria o ensino voltado para o estímulo da higienização, fundamentado na importância das mãos como veículo de contaminação cruzada (indicado por 93,7\% na Tabela 1), sem dar o status de "técnica" necessário na assistência à saúde, mas como continuísmo do ato doméstico que o aluno certamente já teria aprendido? Mais uma hipótese que precisa ser melhor investigada, na direção de compreender a baixa adesão dos PAS à HM .
Os dados referentes ao sabão recomendado para a HM reforçam esta idéia. Apenas $24,6 \%$ dos acadêmicos, especificaram o tipo de sabão recomendado, ou seja, o sabão líquido. E oito $(3,1 \%)$ destes mencionaram a quantidade relativa de sabão necessária para a HM, variando entre 1 - $5 \mathrm{ml}$. A quantidade indicada de sabão líquido para a HM varia de acordo com cada fabricante (4).

A eficácia da HM depende, também, da fricção de todas as superfícies das mãos e dedos (1). A Tabela 2 apresenta as regiões das mãos citadas pelos acadêmicos, ao descreverem de modo sucinto a técnica de HM.

Tabela 2. Distribuição da freqüência e porcentagem das regiões das mãos friccionadas durante a realização da técnica de higienização de mãos segundo acadêmicos de enfermagem $(n=258)$. Goiânia, 2007.

\begin{tabular}{lcc}
\hline Regiões friccionadas & Fr & \% \\
\hline Espaços interdigitais & 188 & 72,9 \\
Punho & 183 & 70,9 \\
Palma com palma & 175 & 67,8 \\
Pontas dos dedos & 170 & 65,9 \\
Palma com dorso & 169 & 65,5 \\
Polegar & 81 & 31,4 \\
Dorso dos dedos & 29 & 11,2 \\
\hline
\end{tabular}

As regiões dos espaços interdigitais $(72,9 \%)$ e punhos $(70,9 \%)$ foram as mais citadas pelos estudantes como sendo aquelas nas quais a fricção é realizada durante a técnica de HM. Em contraste, a região dorsal dos dedos e a região do polegar foram citadas por menos de 50,0\% dos acadêmicos.

No estudo realizado por Scheidt, Carvalho (9), a região menos friccionada pelos PAS foi a ponta dos dedos, esta mesma região em nosso estudo representou 65,9\% das citações as quais os graduandos afirmam friccionar.
A partir destes dados, uma simulação das áreas não friccionadas foi apresentada por meio de figuras identificadas de 1 a 7 , onde o pontilhado representa a área a ser friccionada e a cor cinza a região proporcional a freqüência indicada de fricção. No conjunto os dados mostram que há negligência na realização da técnica de HM pelos acadêmicos, o que para Larson (19) é o principal problema da HM. A autora sugere a seguinte fórmula: impacto da $\mathrm{HM}=$ eficácia $\mathrm{x}$ adesão. 


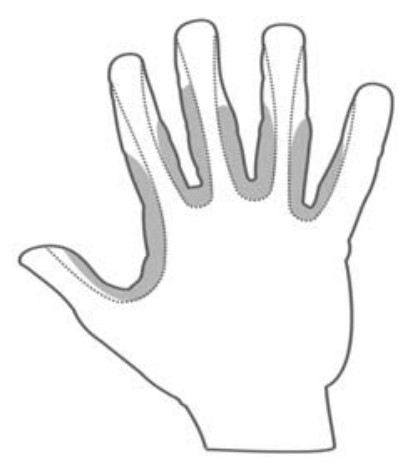

Figura 1. Espaços interdigitais, $188(72,9 \%)$ friccionaram $(n=258)$

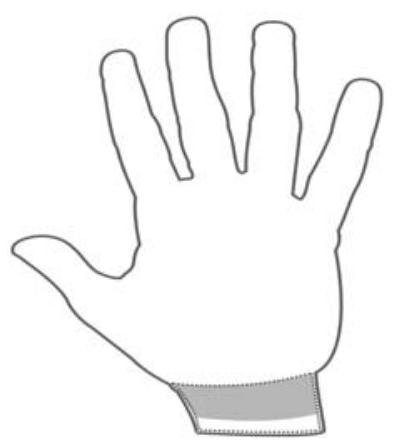

Figura 2. Punhos, $183(70,9 \%)$ friccionaram $(n=258)$

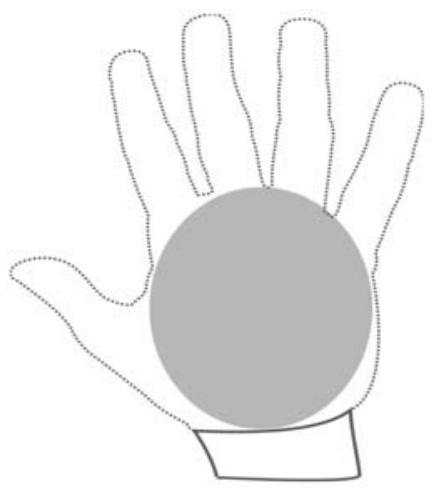

Figura 3. Palma com palma, $175(67,8 \%)$

friccionaram $(n=258)$

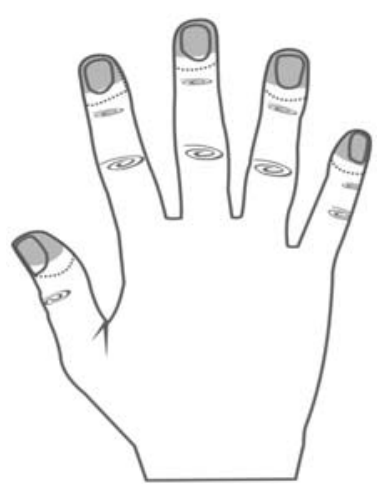

Figura 4. Ponta dos dedos, $170(65,9 \%)$

friccionaram $(n=258)$

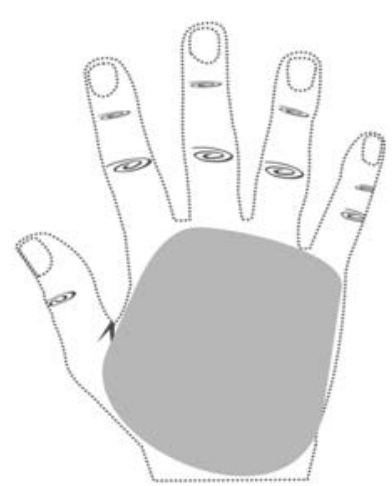

Figura 5. Palma com dorso, $169(65,5 \%)$

friccionaram $(n=258)$

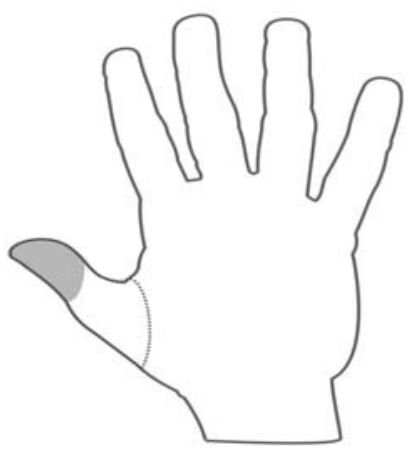

Figura 6. Palma com dorso, $169(65,5 \%)$

friccionaram $(n=258)$

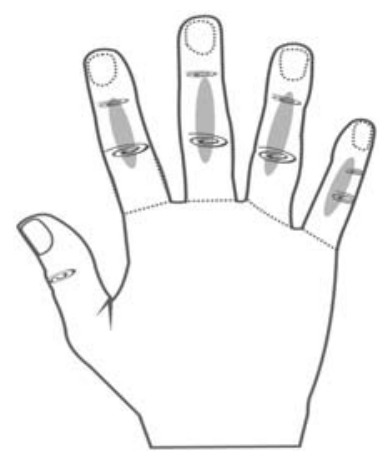

Figura 7. Dorso dos dedos, $29(11,2 \%)$

friccionaram $(n=258)$ 
Analisando o enxágüe das mãos, apenas 98 (38,0\%) acadêmicos destacaram a necessidade de realizar esta etapa respeitando o sentido dos dedos para os punhos que tem a finalidade de evitar a recontaminação (4).

Com relação à indicação do papel toalha para a secagem das mãos conforme as recomendações $(1,4)$, o percentual foi baixo $(56,2 \%)$. No estudo realizado Cardoso et al. (20) com membros da equipe de enfermagem, responsáveis pela administração de medicamentos injetáveis, em um hospital de ensino da cidade de Goiânia - GO, a adesão ao papel toalha foi alta $(93,2 \%)$. Neste caso é preciso lembrar que a adesão depende da disponibilidade do produto e que o que se esperava do questionamento feito aos alunos era o reconhecimento do insumo recomendado. Foi ainda menor o percentual dos acadêmicos $(5,4 \%)$ que referiram a necessidade de secar as mãos obedecendo a sentido "mãos para os punhos".

De acordo com a ANVISA (4) não se deve tocar diretamente a torneira para fechá-la, ao término da HM caso a torneira seja de acionamento manual deve-se utilizar o papeltoalha ou os cotovelos para fechá-la. Neste estudo apenas 48,9\% dos sujeitos destacaram este item, semelhante ao encontrado por Scheidt, Carvalho (9) e Felix (17) nos quais mais de $50,0 \%$ dos sujeitos utilizaram o papel toalha como barreira no fechamento da torneira.

Apenas 25 (9,6\%) acadêmicos descreveram a técnica de HM de forma correta. Ao relacionar este dado com o encontrado na Tabela 1, nos deparamos com um contrasenso, pois apesar de que mais de 90,0\% dos acadêmicos afirmarem ter participado de alguma atividade formal de ensino com relação à técnica de HM, apenas 9,6\% descreveram esta técnica corretamente. Isso nos leva a questionar como este tema tem sido abordado pelas IES. Seria falha na apresentação da técnica de HM ou a ferramenta pedagógica utilizada para o seu ensino não tem sido eficaz?
Os resultados de estudos suscitam mais dúvidas a este respeito, enquanto Mendonça et al. (21), em uma pesquisa com PAS de uma unidade de terapia intensiva neonatal concluiu que $70,6 \%$ dos profissionais realizaram a técnica correta, Scheidt e Carvalho (9) entre PAS encontraram um percentual de $14,0 \%$, e Felix (17), com graduandos de enfermagem apenas 8,8\% e no estudo de Cardoso et al. (20) identificaram que apenas um profissional da equipe de enfermagem $(n=$ 212) que realizou a técnica adequadamente.

A realização da técnica correta seguindo todos os passos recomendados depende em parte da disponibilidade de recursos, entretanto essencialmente depende do "ator" da ação.

\section{CONCLUSÃO}

Segundo os relatos de acadêmicos de enfermagem as IES não têm abordado todos os enfoques sobre HM de maneira satisfatória, merecendo maior atenção os recursos materiais necessários e a indicação do uso do álcool a $70 \%$.

Os acadêmicos apesar de afirmarem ter conhecimento teórico sobre a técnica de HM apresentaram baixo desempenho ao descreverem a técnica, apenas $25(9,6 \%)$ a fizeram de forma correta.

Os resultados mostram que a abordagem feita pelas IES com relação à técnica de HM, não foram suficientes para que o acadêmico descrevesse os passos de realização da técnica, apesar destes dados não permitir afirmar que o acadêmico não executa a técnica de forma correta pode-se inferir que o seu ensino não foi capaz de proporcionar a sedimentação do conhecimento.

Considera-se necessário que as IES invistam no ensino da prevenção e controle de infecção, quem sabe inovando suas práticas pedagógicas. Outro aspecto a considerar é a relação teoria prática, o ensaio realizado por 
Tipple et al. (12) apresenta algumas sugestões e dentre elas consideram que o ensino e a supervisão das medidas de prevenção e controle de infecção devem acontecer de acordo com a necessidade e o momento acadêmico do aluno. E, se pensarmos na técnica de HM que é usualmente apresentada no primeiro ano da graduação, sua aplicação será ao longo de toda a graduação e vida profissional, o que nos leva a compreensão de que todos os docentes devem envolver-se na construção deste conhecimento o que pode ser feito pelo seu exemplo e supervisão.

Concordamos com a ANVISA (4) ao ressaltar a necessidade de colaboração das universidades no desenvolvimento de novos estudos para aumentar o conhecimento sobre a temática e com a finalidade de incorporação de novos projetos pedagógicos que vislumbrem uma nova forma de ensinar esta importante medida nos cursos da área de saúde, contribuindo com a formação de profissionais conscientes e acarretando uma possível mudança no comportamento destes.

\section{REFERÊNCIAS}

1. Apecih. Associação Paulista de Estudos e Controle de Infecção Hospitalar. Guia para higiene de mãos em serviços de assistência à saúde. São Paulo (Brasil): APECIH, 2003.

2. Rocha LA, Borges LFA, Filho PPG. Falta de adesão à lavagem de mãos, ação irritante do uso de sabão e luvas e sua influência na microbiota qualitativa e quantitativa das mãos de enfermeiros. New Lab. 2007; 82: 114-22.

3. Anvisa. Agência Nacional de Vigilância Sanitária. Manual de Higienização das Mãos em Serviços de Saúde. Brasília (Brasil): Ministério da Saúde, 2007.

4. Anvisa. Agência Nacional de Vigilância Sanitária. Manual de segurança do pa- ciente: Higienização das Mãos. Brasília (Brasil): Ministério da Saúde, 2008.

5. Boyce JM, Pittet D. Guideline for hand hygiene in health-care settings: recommendations of the healthcare infection control practices advisory committee and the HICPAC/ SHEA/ APIC/ IDSA Hand hygiene task force. CDC. 2002; 51: 21.

6. Boletim Informativo do Ministério da Saúde. Programa de controle de infecção hospitalar. Lavar as mãos: informações para profissionais de saúde. Brasília (Brasil): Ministério da Saúde, 1989.

7. Portaria no 2616, de 12 de maio de 1998. Normas para o programa de controle de infecção hospitalar. Brasília (Brasil): Ministério da Saúde, 1998.

8. Martini AC, Dall' Agnol CM. Por que lavar ou não as mãos? Motivos de um grupo de enfermagem. R Gaúcha Enferm. 2005; 26 (1): 88-101.

9. Scheidt KLS, Carvalho M. Avaliação prática da lavagem das mãos pelos profissionais de saúde em atividades lúdicoeducativas. R Enferm UERJ. 2006; 14(2): 221-5.

10. Cruz EDA, Pimenta FC, Palos MAP, Silva SRM, Gir E. Higienização de mãos: 20 anos de divergências entre a prática e o idealizado. Cienc. enferm. 2009; 15(1): 33-8.

11. Santos, AM. Controle de infecção: necessidade de novos conceitos. R Prática Hospitalar. 2003; 28 (5): 1-4.

12. Tipple AFV, Pereira MS, Hayashida M, Moriya TM, Souza ACS. O ensino do controle de infecção: um ensaio teórico prático. Rev Latino-Am Enferm. 2003; 11 (2): 245-50.

13. Neves ZCP, Tipple AFV, Souza ACS, Pereira MS, Melo DS, Ferreira LR. Hand hygiene: the impact of incentive strategies on adherence among healthcare workers from a newborn intensive care unit. Rev Latino-Am Enferm. 2006; 14(4):546-52.

14. Tipple AFV, Mendonça KM, Souza ACS, Pereira M, Santos SLV. Higienização das 
mãos: o ensino e a prática entre graduandos na área da saúde. Acta Sci Health Sci. 2007; 29(2): 107-14.

15. Centers for Disease Control and Prevention. Epiinfo program version 3.3 out. 2004. Hallado em: http:// www.cdc.gov/ epiinfo [Acesso 2 agosto de 2008].

16. Conselho Nacional de Saúde. Resolução 196/96 sobre pesquisa envolvendo seres humanos. Brasília (Brasil): Ministério da Saúde, 1996.

17. Felix, CCP. Avaliação da técnica de lavagem de mãos executada por alunos do curso de graduação em enfermagem [dissertação]. Ribeirão Preto (SP). Universidade de São Paulo/USP, 2007.

18. Hayes RA, Trick WE, Vernon MO, Nathan C, Peterson BJ, Segreti J, et al. Ring use as a risk factor (RF) for hand colonization in a surgical intensive care unit (SICU). In: Program and abstracts of the 41st Interscience Conference on Antimicrobial Agents and Chemotherapy, 2001. p.16-19.

19. Larson, EL. Special problems in antisepsis. In: Rutala, WA. Disinfection, Sterilization and Antisepsis: principles, practices, challenges and new research. Washington: Association for Professionals in Infection Control and Epidemiology. 2004. p.104-6.

20. Cardoso SR, Pereira LS, Souza ACS, Tipple AFV, Pereira MS, Junqueira ALN. Antisepsia para administração de medicamentos por via endovenosa e intramuscular. Rev Eletr Enferm. 2006; 8(1): 75-82.

21. Mendonça AP, Fernandes MSC, Azevedo JMR, Silveira WCR, Souza ACS. Lavagem das mãos: adesão dos profissionais de saúde de uma unidade de terapia intensiva neonatal. Acta Sci Health Sci. 2003; 25(2): 147-53. 